\title{
Perspective
}

PERSPECTIVE Actualité en histoire de l'art

$2 \mid 2011$

Les Pays-Bas

\section{Penser le Dutch Design avec les revues néerlandaises}

Theorizing Dutch Design in Dutch journals and reviews

\section{Frederike Huygen}

Traducteur : Bertrand Abraham

\section{(2) OpenEdition}

\section{Journals}

Édition électronique

URL : http://journals.openedition.org/perspective/842

DOI : $10.4000 /$ perspective. 842

ISSN : 2269-7721

Éditeur

Institut national d'histoire de l'art

Édition imprimée

Date de publication : 31 décembre 2011

Pagination : 820-823

ISSN : 1777-7852

Référence électronique

Frederike Huygen, «Penser le Dutch Design avec les revues néerlandaises », Perspective [En ligne], 2 | 2011, mis en ligne le 30 juin 2013, consulté le 01 octobre 2020. URL : http://journals.openedition.org/ perspective/842; DOI : https://doi.org/10.4000/perspective.842 


\section{Penser le Dutch design avec les revues néerlandaises}

\section{Frederike Huygen*}

L'ensemble du monde artistique néerlandais est actuellement dans l'attente des réductions draconiennes de crédits et de subventions qui le menacent, et qui auront à coup sûr des incidences sur les revues et les magazines. Les plus substantiels d'entre eux ont toujours occupé une position marginale sur le marché, car ils touchent un lectorat limité du fait de l'exiguïté de l'aire linguistique néerlandaise. La publication de nombreuses revues artistiques est donc étroitement dépendante de subventions et de fonds extérieurs. Une analyse des différentes publications ne peut éluder ce facteur économique, qui revêt en outre d'autres aspects, tels que le manque d'annonceurs et la faible rémunération des auteurs.

Un second facteur à prendre en compte dans l'étude des revues concerne le niveau et la situation de la critique en matière d'art, d'architecture et de design. À une époque où le déclin des médias imprimés s'accélère, cette critique dépérit ou se voit, elle aussi, marginalisée. En outre, un changement s'est opéré durant la dernière décennie quant au type d'informations véhiculées par les médias dans ces domaines. Pour ce qui est de la critique portant sur le design, on assiste depuis des années à une érosion du contenu due au fait que son objet est désormais en partie liée avec le mode de vie des consommateurs et avec les gadgets. De telles évolutions ne sont pas spécifiques aux Pays-Bas et peuvent être observées à l'échelle internationale, comme le montre un numéro spécial de la revue Oase $\left(n^{\circ} 81\right.$, 2010) consacré à la critique architecturale, dans lequel s'expriment des auteurs allemands, français, belges et finlandais. Chose étrange, ces évolutions n'ont apparemment aucun effet sur le marché du livre qui, à en juger par l'offre croissante des publications, semble florissant. Bien que la plupart des éditeurs de livres d'art entendent prendre le moins de risques possible et qu'ils soient, eux aussi, très dépendants des subventions, ils publient une quantité relativement importante d'ouvrages consacrés aux designers et aux architectes, ainsi que des livres traitant de projets spécifiques. Recherche d'une mise en forme expressive et recours à la photographie sont souvent les caractéristiques dominantes de ces publications. Dans le présent article, il convient cependant de faire abstraction des livres et des magazines de luxe et d'art de vie pour concentrer notre attention sur les revues professionnelles consacrées au design et, à un moindre degré, à l'architecture.

\section{Approches généralistes du Dutch Design}

Une des plus anciennes revues entièrement consacrées au design, Items, a presque trente ans d'existence (fig. 1). Conçue en 1982 à l'initiative d'étudiants en architecture à la Technische Universiteit Delft, elle a longtemps été une affaire d'amateurs fonctionnant grâce à des auteurs généreux, des jeunes gens dynamiques et un imprimeur charitable. Elle a notamment servi de plate-forme à de jeunes designers qui envoyaient de leur propre chef des contributions. Peu à peu, une ligne rédactionnelle plus cohérente s'est affirmée à travers des numéros thématiques et une large palette de sujets. Au sein de la rédaction, une légère préférence pour l'architecture high-tech et l'esthétique géométrique moderniste s'est imposée, ainsi qu'un intérêt appuyé, dans les années 1980, pour le groupe de designers néerlandais résolument indépendant qui s'est constitué dans le sillage de l'influent Groupe de Memphis (fondé en Italie en 1980 par Ettore Sottsass).

L'approche très large de la discipline demeure le propre de cette revue, en dépit des changements de rédacteurs en chef et d'éditeurs. Le contenu actuel englobe graphisme, design industriel, design social, mode et arts appliqués, et l'un des six numéros annuels d'Items est invariablement consacré aux examens de fin d'étude des écoles de design. La revue propose à ses lecteurs des articles, des interviews, des " portfolios " d'artistes, des annonces
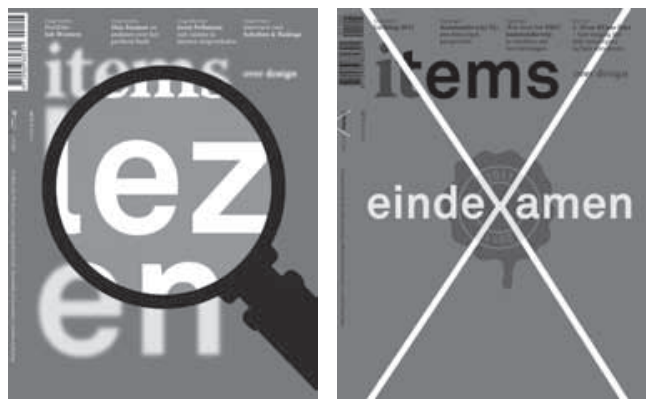
de parution, des actualités et une rubrique de critiques de livres. Elle organise également des rencontres et dispose depuis peu d'un site Internet (www.items.nl). Les contributions sont en général davantage des textes d'information que d'opinion, la revue ne cherchant pas, en tout état de cause, à suivre à la trace l'ensemble de l'actualité, ni à être un média ultra-spécialisé.

Si c'est là un avantage pour les lecteurs souhaitant rester informés de façon globale, un public en quête d'informations spécifiques, par exemple sur le graphisme ou l'artisanat, y trouvera moins son compte. Néanmoins, deux autres revues néerlandaises proposent, chacune à sa manière, des approches plus ciblées. D'ordre technique, le magazine Product est dédié aux nouveaux matériaux et aux nouvelles technologies en lien avec le design industriel. Un autre support ponctuel mais important est la revue Metropolis $M$. Consacrée à l'art moderne et proposant également des articles en anglais, elle publie régulièrement des analyses qui passent au crible les évolutions du Dutch design. Une pensée critique d'un niveau élevé, et un certain registre intellectuel et théorique distinguent cette revue.

Dans le domaine de l'architecture, De Architect est comparable à Items : informative, variée, attentive aux évolutions et offrant des comptes rendus d'actualités. Deux des numéros annuels sont consacrés à l'architecture d'intérieur et mettent en valeur des créateurs de design et des projets spécifiques. Ainsi, dans le numéro paru en mars 2011, le Salon du meuble à Milan et les objets créés par le plasticien Joep van Lieshout étaient à l'honneur. À la différence de Metropolis $M$, qui s'interroge sur des phénomènes pointus comme la mode des exemplaires uniques hors de prix destinés aux happy few, De Architect répand l'évangile du design néerlandais. Elle promeut notamment l'idée que le succès des designers néerlandais à travailler en collaboration avec des industries à l'international serait le fait de la disposition d'esprit singulière des Néerlandais. Si la réussite du Dutch Design est certes intéressante et mérite d'être signalée, la répétition d'idées reçues de ce genre est parfois lassante. Publié en édition bilingue (néerlandais et anglais), De Architect dispose également d'un riche site Web (www.dearchitect.nl) qui met en avant dossiers, thèmes, projets et produits.

\section{Politiques du design}

La revue Morf est d'un tout autre ordre. Elle est éditée par Premsela, l'Institut néerlandais pour le design et la mode à Amsterdam, qui soutient des initiatives existantes, co-organisant des expositions et des débats tout en développant ses propres activités. Morf paraît deux fois par an depuis 2004, sous la forme d'un petit livre maniable, et est distribué gratuitement aux étudiants des filières design. L'idée maîtresse de la revue est de republier des textes anciens mais importants sur le design défini dans le sens le plus large du terme. Elle encourage également la participation d'étudiants, dont les contributions sous forme d'essais accompagnent les textes principaux. Les designers étant généralement des gens d'action formés surtout à l'image - la plupart des formations ne proposent aucun enseignement en histoire du design -, une des vocations de Morf est de les inciter à acquérir une conscience historique de leur discipline ainsi que des habitudes de lecture. Les textes publiés dans la revue sont de natures fort différentes, relevant de sources, de périodes historiques et d'aires géographiques extrêmement diverses, et rassemblés, du moins pour les derniers numéros, sous un thème commun. La parution $n^{\circ} 13$ d'automne 2010 (fig. 2) explore ainsi les rapports entre politique et design, développés à partir de la relation entre maillot de bain et féminisme, la diffusion de la machine à coudre dans les campagnes indiennes initiée par Gandhi, ou encore l'exportation massive des tissus imprimés « africains " par l'entreprise néerlandaise Vlisco. Les textes sont brièvement présentés et leur auteur fait parfois l'objet d'une notice biographique. Morf est diffusé en libraire depuis peu.

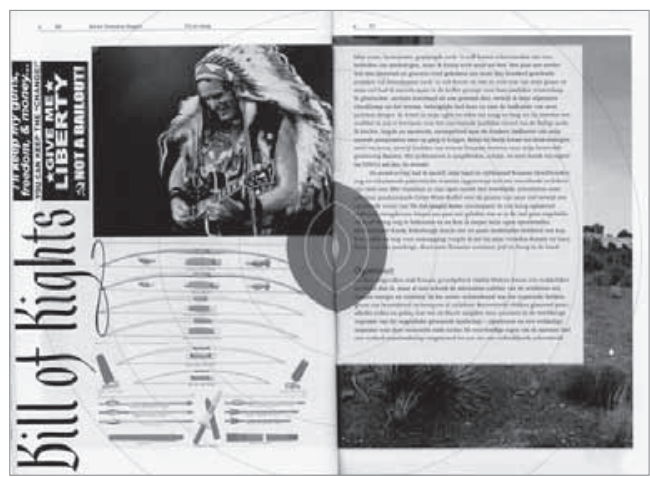

2. Pages intérieures de Morf, 13, 2010, consacré au thème « Design/ Politiek ». 
3. Couverture de Oase, 69, 2006, "Positions », créée par Karel Martens et Layla Tweedie-Cullen.

4. Couverture de Volume, 27, avril 2011, consacré au vieillissement.
D'autres revues encore, également dignes d'intérêt, occupent des niches spécifiques du marché. Les cahiers Open et Boekman, par exemple, partagent le même souci de mettre en lumière, sur la place publique, des questions comme celles de la tolérance, du populisme, de la vie privée, ou de l'influence des nouveaux médias. Elles constituent toutes les deux un forum de discussion, de débat, de réflexion et de construction théorique, auquel scientifiques et artistes apportent leur concours. S'opère de la sorte une pollinisation croisée entre sociologues, politologues et penseurs d'autres disciplines, à propos de l'art, des affaires publiques et des évolutions actuelles de la société. Dans Open, éditée par la SKOR (Fondation pour l'art dans l'espace public), des artistes réagissent sur ces sujets par des contributions sous forme d'images et parfois de textes. S'il présente de nombreux points communs avec Open, Boekman est un peu plus axé sur la politique artistique et culturelle du fait qu'il est édité par la Fondation Boekman, structure spécialement créée pour investir ce domaine. Art et commerce, culture "populaire " ou " d'élite ", art et engagement, art communautaire, le métier d'artiste sont parmi les thèmes traités dans ses pages. Les deux revues accordent une place à la critique de livres et elles se ressemblent sur le plan visuel : même petit format A5, même volonté de privilégier le texte par rapport à l'image. Open paraît également en anglais.

Open et Boekman confèrent au débat néerlandais sur l'art et la culture une densité qui lui est indispensable. L'intelligence et l'à-propos avec lesquels elles soulèvent les problèmes, et l'impulsion considérable et la qualité qu'elles apportent au débat font de ces deux revues des publications irremplaçables pour les questions sur les arts et la société. Boekman constitue de surcroît des dossiers thématiques, dans lesquels sont rassemblées des références bibliographiques et des adresses Internet utiles. Toutefois, malgré l'essor de pratiques telles que le «design social», peu de designers interviennent à l'écrit dans ces débats ou se

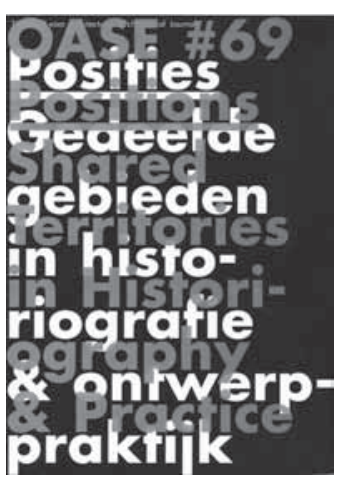

manifestent par des analyses solides et des contributions. L'absence des praticiens de l'arène de la discussion théorique est non seulement regrettable mais peut être aussi un signe de l'impuissance de la profession elle-même, en dépit du fait qu'elle ne cesse de s'afficher comme étant engagée et concernée. Elle a beau paraître consciente de toutes sortes de problèmes et de questions, elle est, tout compte fait, encore peu apte à jouer un rôle théorique véritablement important.

\section{De la théorie à la pratique : vers le design thinking}

La petite revue Oase entend se situer au juste milieu entre l'argumentation théorique universitaire et la pratique. Ce cahier en anglais et en néerlandais, dirigé depuis trente ans par un groupe de bénévoles très engagés appuyés par des auteurs et des amis, entend susciter, dans le domaine de l'architecture, des questions sur le design, la critique, l'histoire et la théorie (fig. 3). Il publie, à cet effet, trois numéros thématiques par an mettant en lumière des projets choisis. Dans la rétrospective d'ensemble que propose le numéro 75 de 2008, Maarten Delbeke définit Oase comme un "lieu de rencontre entre une conception concrète, disciplinaire de l'architecture, correspondant à celle qu'ont la plupart des architectes en exercice, et la conception historicoesthétique de la critique spécialisée " ${ }^{1}$.

Alors qu'Oase part du principe que l'architecture constitue une discipline spécifique, la position de la revue Volume (fig. 4) consiste à affirmer qu'elle touche, au fil de ses développements, un champ beaucoup plus large d'activités intellectuelles et de recherches. Volume, revue fondée en 2005 et entièrement rédigée en anglais, est une émanation de

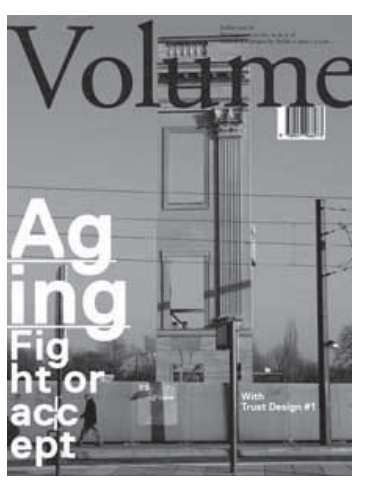

la Fondation Archis pour les actions spatiales et culturelles, et collabore au collectif OMA (Office for Metropolitan Architecture) fondé par Rem Koolhaas. Cette revue est, elle aussi, thématique dans sa conception, et combine articles 
théorico-critiques et présentations de projets. Le $n^{\circ} 27$ paru en avril 2011 est entièrement consacré au vieillissement, et contient aussi bien des reportages sur Benidorm et la Floride que des articles sur la restauration et la reconstruction de bâtiments, et des présentations sur des projets des villes ou sur le logement des personnes âgées. Dans le même numéro se trouve également un supplément sériel (réparti dans quatre publications) intitulé « Trust Design " sur la notion de la confiance qu'inspire le design, à quoi s'ajoute un rapport sur la façon dont elle a été mise en œuvre par les étudiants de la Design Academy d'Eindhoven. Théorie et recherche sont ici encore en contact étroit avec la pratique du design elle-même.

Cette symbiose correspond à une tendance actuelle, qui consiste à caractériser de plus en plus cette pratique en même temps qu'elle reflète une évolution en direction du design social ${ }^{2}$. Développement durable, environnement, santé et collectivité deviennent des sujets de prédilection pour les designers, qu'ils soient abordés ou non de façon interdisciplinaire. Cette stratégie du design thinking connaît une progression considérable et transforme la discipline. Aussi difficile qu'il puisse être à ce stade de juger ou d'évaluer ces nouvelles orientations, le changement de paradigme qu'elles entraînent aura sans doute une influence sur nos façons de débattre et d'écrire sur le design.

* Ce texte a été traduit par Bertrand Abraham.

1. «... the place where a concrete, disciplinary conception of architecture, such as that held by most practising architects, meets the historical-aesthetic conception of the specialist critic " (Maarten Delbeke, "Period 4 (1997-2006) ", dans Oase, 75, 2008, p. 256-263, en particulier p. 261.

2. En témoigne un récent numéro de la revue allemande Kunstforum, 207, mars-avril 2011, consacré au Social Design.

Frederike Huygen, Stichting Designgeschiedenis f.huygen@xs4all.nl

Mots-clés : XX'-XIX siècles, architecture et design, édition, graphisme, produits de consommation, théorie du design.

\section{Les revues d'histoire de l'art aux Pays-Bas}

\section{Léonard Pouy}

Les revues d'histoire de l'art néerlandaises sont aujourd'hui dans une situation critique, et ce à plus d'un titre. Certes, le prestige durable de certaines revues d'art ancien continue de rayonner sur le paysage éditorial scientifique des Pays-Bas, tandis que les revues universitaires, se distinguant par une tendance à la pluridisciplinarité, enrichissent la recherche en histoire de l'art. Force est toutefois de constater que la situation éditoriale a considérablement évolué ces dernières années. Elle se caractérise principalement par une forte diminution du nombre des publications ${ }^{1}$ dans un contexte de mutation profonde de la politique culturelle étatique, marquée par de fortes restrictions budgétaires et par la promotion de l'esprit d'entreprise ${ }^{2}$.

Dans ce contexte perturbé, certains centres d'activité éditoriale s'imposent. La ville d'Amsterdam jouit naturellement de la présence de nombreux musées qui éditent des bulletins, des chroniques et des études, mais elle domine également ce paysage par ses revues étudiantes qui révèlent certaines innovations de la recherche et s'ouvrent régulièrement à d'autres disciplines. La ville de La Haye conserve, quant à elle, son statut de capitale grâce à la présence, outre de musées prestigieux et de marchands importants, du Rijksbureau voor Kunsthistorische Documentatie (RKD), fondé en 1932 et fédérant à lui seul une large part de l'activité scientifique néerlandaise concernant l'histoire de l'art. La revue Oud Holland, anciennement éditée à Amsterdam, pourrait ainsi être qualifiée aujourd'hui de haguoise étant donné ses liens avec la Mauritshuis et le RKD. Ce dernier, qui héberge également la revue Delineavit $\theta$ Sculpsit, publie d'ailleurs son propre bulletin, créé en 1993 par la toute jeune Fondation Hofstede de Groot et par les amis du $\mathrm{RKD}^{3}$. À Utrecht, la célèbre revue Simiolus, liée à l'université, a pour sa part largement diffusé, à partir des années 1960, les recherches iconologiques et les nouvelles théories propres à son 